\title{
Sodium Glucose Transporter 2 (SGLT2) Inhibition does not Protect the Myocardium from Acute Ischemic Reperfusion Injury but Modulates Post- Ischemic Mitochondrial Function
}

\author{
Jespersen NR ${ }^{*}$, Lassen TR, Hjortbak MV, Støttrup NB and Bøtker HE \\ Department of Cardiology, Aarhus University Hospital, Aarhus, Denmark
}

*Corresponding author: Nichlas Riise Jespersen, Department of Cardiology, Aarhus University Hospital, Palle Juul-jensens Boulevard 99, 8200 Aarhus N, Denmark, Tel: +45 30254287; E-mail: n.riise.jespersen@clin.au.dk

Received date: April 03, 2017; Accepted date: April 26, 2017; Published date: April 29, 2017

Copyright: (c) 2017 Jespersen NR, et al. This is an open-access article distributed under the terms of the Creative Commons Attribution License, which permits unrestricted use, distribution, and reproduction in any medium, provided the original author and source are credited.

\section{Abstract}

Background: The Sodium Glucose Transporter 2 (SGLT2)-inhibitor, empagliflozin, reduces death from cardiovascular causes. We hypothesized that the mechanism involved direct protection against IschemiaReperfusion (IR) injury and improved post-ischemic mitochondrial function.

Methods: We examined infarct size (series I) and mitochondrial respiration (series II) in four groups of isolated perfused hearts from male Wistar rats: Sham-operated hearts (Sham group), IR-injured hearts (IR group), hearts treated with ischemic preconditioning (IPC) by $2 \times 5 \mathrm{~min}$. cycles of IR prior to sustained ischemia (IPC group), and hearts co-perfused with $2.14 \mathrm{mg} / \mathrm{l}$ of empagliflozin $10 \mathrm{~min}$. prior to sustained ischemia (EMPA group).

Results: In contrast to IPC, empagliflozin did not reduce infarct size compared to the IR group, when given 10 min prior to the acute myocardial infarction. Empagliflozin improved post-ischemic complex I+II respiration compared to the IR group. This improvement was similar to IPC. In contrast to the improved complex I respiration by IPC, empagliflozin mainly improved complex II respiration. Empagliflozin hearts had significantly higher respiration in oligomycin induced state 4 than the sham and IR group, indicating that empagliflozin modulates the inner mitochondrial membrane.

Conclusion: In conclusion, empagliflozin yielded no acute cardioprotection in the isolated perfused non-diabetic rat heart. Empagliflozin mainly improved complex II respiration and increased permeability of the inner membrane, providing a potential explanation for the positive long-term effects observed in post infarction myocardial dysfunction.

Keywords: Ischemic-reperfusion injury;
inhibition; Cardioprotection; Mitochondrial respiration

\section{Introduction}

The Sodium Glucose Transporter 2 (SGLT2)-inhibitor, empagliflozin, reduces heart failure and cardiovascular mortality among type 2 diabetes mellitus patients in the absence of a simultaneous reduction in nonfatal myocardial infarction or nonfatal stroke. Hence, the benefit of empagliflozin seems to be caused by improvement in patients, who experience a cardiovascular event rather than in the prevention of atherosclerotic events [1]. Empagliflozin by itself or through shift in substrate supply towards energy efficient $\beta$ hydroxybutyrate [2] may induce protection against Ischemic Reperfusion (IR) injury during an acute myocardial infarction.

Preservation of mitochondrial function and integrity is of major importance for the outcome following acute ischemia. During ischemia, lack of ATP leads to $\mathrm{Ca} 2+$ overload, mitochondrial dysfunction and ultimately cell death. Initiation of reperfusion is essential to salvage the tissue but by itself induces further damage to the cells due to oxidative stress originating from the mitochondria. This leads to impaired post-ischemic mitochondrial function resulting in a worsened outcome [3]. We hypothesized that empagliflozin per se induces cardioprotection directly on the heart by improved postischemic mitochondrial performance.

\section{Methods}

Male Wistar rats (8-9 weeks old, 300-350 g, Janvier, France) were given time to acclimatize after transportation at a constant temperature of $23^{\circ} \mathrm{C}$ with a $12 \mathrm{~h}$ light-dark cycle and allowed unlimited access to food and water. Rats were handled according to national guidelines in Denmark and the Guide for the Care and Use of Laboratory Animals published by the National Institute of health (http://www.nap.edu |readingroom $\mid$ books $\backslash$ labrates $\backslash$ ). The study conformed to Danish law for animal research (Act no. 1306 of 23/11/2007, Danish Ministry of Justice).

We compared infarct size (series I) and mitochondrial respiration (series II) in 4 groups ( $\mathrm{n}=7-8)$ : Sham-operated hearts (Sham group), IR-injured hearts (IR group), IR-injured hearts exposed to ischemic preconditioning (IPC) by $2 \times 5 \mathrm{~min}$, cycles of IR prior to sustained global no-flow ischemia (IPC group), and IR-injured hearts coperfused with $2.14 \mathrm{mg} / \mathrm{l}$ of empagliflozin $10 \mathrm{~min}$, prior to global noflow ischemia (EMPA group). The concentration of empagliflozin was calculated from the maximal peak plasma concentration used in clinical practice. 
Citation: Jespersen NR, Lassen TR, Hjortbak MV, Støttrup NB, Bøtker HE (2017) Sodium Glucose Transporter 2 (SGLT2) Inhibition does not Protect the Myocardium from Acute Ischemic Reperfusion Injury but Modulates Post-Ischemic Mitochondrial Function. Cardiovasc Pharm Open Access 6: 210. doi:10.4172/2329-6607.1000210

Page 2 of 3

Hearts were isolated and perfused using an ex-vivo heart model as previously described [4]. The hearts were perfused with a KrebsHenseleit buffer containing glucose $(11 \mathrm{mmol} / \mathrm{L})$ and stabilized for 40 min, followed by $40 \mathrm{~min}$ of global no-flow ischemia. In the infarct studies, a total of $120 \mathrm{~min}$ of reperfusion was given before infarct staining with $1 \%$ Triphhenyltetrazoliumchloride. In the mitochondrial studies, a total of $30 \mathrm{~min}$ of reperfusion was used, before the left ventricle was removed and cooled.

Mitochondrial respiration was measured with high-resolution respirometry (Oroboros, Innsbruck, Austria) in permeabilized cardiac muscle fibers as previously described [5]. In short, two titration protocols were used to evaluate glucose and fatty acid linked respiration: Protocol 1 (complex I+II-linked respiration): State 2 respiration $(\mathrm{GM})$ was assessed with malate $(2 \mathrm{mM})$ and glutamate $(10$ $\mathrm{mM})$. Following addition of $\mathrm{ADP}(5 \mathrm{mM})$, state 3 respiration in complex I was evaluated (GM3)

Next cytochrome c $(10 \mu \mathrm{M})$ was added to control for outer mitochondrial membrane integrity (more than $10 \%$ increases in respiration leads to exclusion). Subsequently, Succinate $(20 \mathrm{mM})$ was added to activate complex II during state 3 (GMS3) followed by Oligomycin $(2.5 \mu \mathrm{M})$ to test inner mitochondrial integrity by achieving state 4 respiration (4o). Finally Rotenone $(0.5 \mu \mathrm{M})$ and Antimycin A $(2.5 \mu \mathrm{M})$ were added to estimate residual oxygen consumption (ROX). Protocol 2 (fatty acid oxidation): Malate $(2 \mathrm{mM})$ was added and Octanoyl-1-carnitine $(0.2 \mathrm{mM})$ was titrated in steps to examine $\beta$ oxidation at state $2(\mathrm{MOc})$. State 3 respiration (MOc3) was achieved by adding ADP $(5 \mathrm{mM})$ and finally Cytochrome $\mathrm{C}(10 \mu \mathrm{M})$ was added to examine outer membrane integrity.

All results are presented as means \pm SEM. Infarct size and mitochondrial respiration measurements were analyzed by one-way ANOVA with turkey's post-test. P-values $<0.05$ were considered significant. All statistical analyses were performed using GraphPad Prism version 5.0 software (GraphPad Software, La Jolla, CA).

\section{Results}

Empagliflozin did not reduce infarct size compared to the IR group when given $10 \mathrm{~min}$ prior to myocardial ischemia. IPC reduced the infarct size compared to both the IR group and EMPA group (Figure 1A).

Similar to IPC, empagliflozin improved post-ischemic complex I+II respiration (GMS3) compared to IR group, even though respiration in complex I (GM3) alone was significantly lower in empagliflozin hearts than in IPC hearts, reflecting an improvement of complex II function (Figure 1B).

Empagliflozin induced a significantly higher respiration in state 4 by oligomycin (4o) than the Sham and IR groups. Hence, empagliflozin permeabilized and uncoupled the inner mitochondrial membrane. Empagliflozin did not protect mitochondrial fatty acid respiration (MOc3) when compared to IR group.

\section{Discussion}

We found no acute cardioprotective effect of empagliflozin on infarct size in the non-diabetic isolated perfused rat heart. However, post-ischemic mitochondrial function was affected by administration of empagliflozin with improved complex I+II respiration and increased uncoupling of the inner membrane. The modulation was evident in the absence of $\beta$-hydroxybutyrate as substrate.

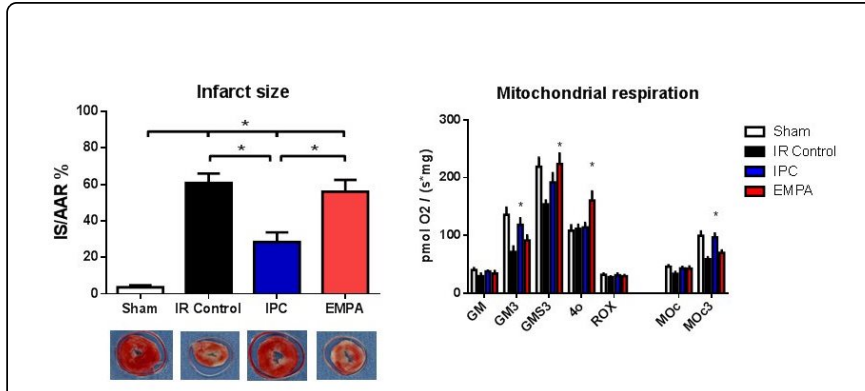

Figure 1: (A) Infarct size as infarct size (IS)/area-at-risk (AAR), Below representative stained heart slices. (B) Mitochondrial respiratory capacity. GM: State 2 respirations with glutamate +malate; GM3: State 3 respirations with glutamate and malate; GMS3: State 3 respirations with glutamate, malate, and succinate; 4o: State 4 respirations with oligomycin; ROX: residual oxygen consumption evaluated after adding rotenone and antimycin $\mathrm{A}$; EMPA=Empagliflozin, IPC: Ischemic preconditioning; IR Control: Ischemic-reperfusion control. Sham group illustrates the influence of the experimental model on myocardial injury and respiration measurements. Data are means \pm SEM. ${ }^{\star} \mathrm{P}<0.05$ vs. IR Control.

Improvement of mitochondrial respiration is of importance in acute ischemia and heart failure. While complex II does not serve as an individual proton pump in the Electron Transport Chain (ETC), it serves as an important regulatory step between the ETC and tricarboxylic acid cycle. Improvement in complex II respiration might be important for the remodeling after an ischemic insult and protect the heart from development of heart failure.

Increased permeability of the inner membrane uncouples mitochondrial respiration and may improve cell survival and mitochondrial respiration [6]. Optimized mitochondrial respiration is of importance in remodeling of infarcted tissue because chronic oxidative stress is attenuated [7]. This mechanism may explain the long term reduction in heart failure observed in the EMPA-REG trial.

We used a non-diabetic rat heart model to examine the genuine cardioprotective effect of empagliflozin independent on the metabolic disarrays inherent to diabetes mellitus. Whether similar results can be extrapolated to the diabetic heart remains a limitation.

\section{Conclusion}

In conclusion, empagliflozin per se does not reduce infarct size in the isolated perfused non-diabetic rat heart. However, empagliflozin modulates post-ischemic mitochondrial function independently of $\beta$ hydroxybutyrate indicating that empagliflozin itself may modulate post-infarction left ventricular remodeling and development of heart failure. Further studies are needed to apprehend the protective properties of empagliflozin.

\section{Acknowledgement}

We sincerely thank Hans Nymand Pedersen and Casper Carlsen Elkjær for their technical assistance. 
Citation: Jespersen NR, Lassen TR, Hjortbak MV, Støttrup NB, Bøtker HE (2017) Sodium Glucose Transporter 2 (SGLT2) Inhibition does not Protect the Myocardium from Acute Ischemic Reperfusion Injury but Modulates Post-Ischemic Mitochondrial Function. Cardiovasc Pharm Open Access 6: 210. doi:10.4172/2329-6607.1000210

Page 3 of 3

\section{Funding}

The present study was supported by grants from the Danish Council for Strategic Research (Innovation fund, 11-115818) and Aarhus University.

\section{References}

1. Zinman B, Wanner C, Lachin JM, Fitchett D, Bluhmki E, et al. (2015) Empagliflozin, Cardiovascular Outcomes, and Mortality in Type 2 Diabetes. N Engl J Med 373: 2117-2128.

2. Ferrannini E, Mark M, Mayoux E (2016) CV Protection in the EMPAREG OUTCOME Trial: A "Thrifty Substrate" Hypothesis. Diabetes Care 39: 1108-1114.

3. Halestrap AP, Clarke SJ, Javadov SA (2004) Mitochondrial permeability transition pore opening during myocardial reperfusion-a target for cardioprotection. Cardiovasc Res 61: 372-385.
4. Stottrup NB, Lofgren B, Birkler RD, Nielsen JM, Wang L, et al. (2010) Inhibition of the malate-aspartate shuttle by pre-ischaemic aminooxyacetate loading of the heart induces cardioprotection. Cardiovasc Res 88: 257-266.

5. Jespersen NR, Yokota T, Stottrup NB, Bergdahl A, Paelestik KB, et al. (2017) Pre-ischaemic mitochondrial substrate constraint by inhibition of malate-aspartate shuttle preserves mitochondrial function after ischaemia-reperfusion. J Physiol.

6. Teshima Y, Akao M, Jones SP, Marban E (2003) Uncoupling protein-2 overexpression inhibits mitochondrial death pathway in cardiomyocytes. Circ Res 93: 192-200.

7. Vasquez TC, Garcia CI, Pennanen C, Parra V, Hill JA, et al. (2016) Mitochondrial dynamics, mitophagy and cardiovascular disease. J Physiol 594: 509-525. 